\title{
Different surgical treatment of post-burn contracture of foot and ankle joint
}

\author{
Shakirov Babur \\ Samarkand State Medical Institute, Burn department of RSCUMA and Inter-Regional Burn Center, Samarkand, Uzbekistan
}

Email address:

baburshakirov@yahoo.com

\section{To cite this article:}

Shakirov Babur. Different Surgical Treatment of Post-Burn Contracture of Foot and Ankle Joint. Journal of Surgery. Vol. 1, No. 2, 2013, pp. 32-36. doi: 10.11648/j.js.20130102.16

\begin{abstract}
The foot and ankle scar post burn contractures are the result of deep partial and full-thickness burns that affect foot motion, impair the lower extremity function and benefit from surgical reconstruction. 76 patients (ages 2-46) with post burn foot and ankle joint contractures were treated at the Inter-regional Burn Center and burn department of RCSUMA Samarkand, Uzbekistan. The operations must be performed within 6-12 months after healing of burn wounds to prevent secondary changes. In the case when contracture is severe, the operation must be performed as soon as possible. The method of operation should be chosen according to both severity and localization of the injury, using local uninjured tissues and soft scars to make trapezoid, Z-form, triangular, free and other grafts placed on the area of the excised scars Results in $71,05 \%$ of all cases the contracture of the foot-ankle joint was completely eliminated and the conditions necessary for the rehabilitation of the injured ankle were ensured. There was improvement after burn treatment or secondary changes due to inflammatory processes and foot inactivity due to its wrong position; in 3,95\% there was not improvement. Negative results were due to deep tissue defect, irreversible changes in the bone- joints, late recourse to medical aid, and post operative effect. We did not observe any ulceration of skin grafts.
\end{abstract}

Keywords: Post Burn Foot, Ankle Contracture and Surgical Treatment

\section{Introduction}

The foot and the ankle joint represent 5 to $7 \%$ of all postburn deformities [1]. The foot and ankle scar post burn contractures are the result of deep partial and full-thickness burns that affect foot motion, impair the lower extremity function and benefit from surgical reconstruction. Functional disturbances of the foot and ankle joint may severely affect posture and gait, and can even lead distortion of the pelvis, curvature of the spine [2]. The dorsum of the foot and the ankle areas are injured more often [3].

Contact with burning sandal woods typically causes these burns $[4,5]$. Sandal burns are characterized by such severe deep injuries because of a close contact of the body with live coals or woods and include not only skin injuries of various depths but also injuries to underlying tissues: subcutaneous fat, fasciae, muscles, and even bones.

The scars in the ankle and foot area are prone to keloid growth or become pathological with ulcerations.

Foot and ankle joint contractures are classified into four different degrees in reference to the neutral position of the foot. The amplitude of ankle joint movements is taken into consideration as a basis, normally equal to $65-80^{\circ}$, i.e. 40 $50^{\circ}$ of plantiflexion and $20-30^{\circ}$ - of dorsiflexion. The extent of the contracture is determined in relation to the limitation of movement expressed in degrees.

The experience of the Inter-Regional Burn Center in the rehabilitation and reconstructive surgery of burns suggests that post-burn deformities of extremities represent a separate group of severe orthopedic diseases [6-10].

It has been found out that $75 \%$ of the Burn Center patients who had undergone deep burns need rehabilitation and about $35 \%$ of patients need surgical treatment. Investigators routinely consider these deformities to separate localizations as completed processes isolated from the general burn disease process. The significant number of patients undergoing surgical rehabilitation treatment after burns suggests that the conservative methods of therapy currently used are not effective. There are some studies on different aspects of rehabilitation and reconstruction of post-burn extremity deformations in patients [11-14].

The aim of the present study was to evaluate of different reconstructive surgery techniques in patients with post-burn 
contractures and deformities of feet and ankle joints.

\section{Material and Methods}

Since 1990, 76 patients (ages 2-46) with post burn foot and ankle joint contractures were operated upon personally; 44 were male and 32, female. Eleven patients had contracture on both foot and ankle joint. The cases of the burn were as follows: sandal burns $52 \%$ and other $48 \%$. Among all the cases, 51 were old contractures persisting for 1 - 5 years, while 25 were contractures existing for six - ten years.

First and second degree contractures were observed in 15 cases, third - degree in 42 cases, and fourth - degree in 19 cases. Marked deformities of bone-ankle joint changes were observed at 23 patients, with changes in the form and slow growth of bones in foot ankle with its valgus or varus deformities.

The main types of burn after-effects of foot- ankle joint are presented in the table №1.

Table №1. Type of after effect

\begin{tabular}{cccc}
\hline Position of deformity of patients & Patients & $\begin{array}{c}\text { Feet and } \\
\text { ankles } \\
\text { joint cases }\end{array}$ & $\begin{array}{c}\text { Per/cent } \\
\%\end{array}$ \\
\hline I Isolated contracture of foot-ankle & 28 & 28 & 36,84 \\
joint & 9 & 9 & 11,84 \\
1. Dorsal flexion & 7 & 7 & 9,21 \\
2. Lateral surface & 5 & 5 & 6,58 \\
3. Plantar flexion & 7 & 7 & 9,21 \\
4. Whole ankle joint & 48 & 59 & 63,16 \\
$\begin{array}{c}\text { II Extended contractures of the dorsum } \\
\text { of the foot (digits, ankle joint) } \\
\text { Total }\end{array}$ & 76 & 76 & $100 \%$ \\
\hline
\end{tabular}

\section{Results}

\subsection{Isolated Contracture of Foot-Ankle Contracture}

Scar contracture varies, depending on its location. Using anatomical principles we distinguished the following contractures of foot-ankle joint: dorsiflexion (9 cases), lateral surface (7), plantiflexion (5), and whole ankle joint (7). The main aim is to correct the position of the foot. The type of plastic operation was determined in relation to the location and spreading of the scars, the degree of contracture, and the in act skin located nearby.

Dorsiflexion.

Dorsal contractures of the foot limit plantar flexion. Dorsal scars due to third and fourth degree burns can cause dorsiflexion contractures of the foot and ankle as great as to $30-40^{\circ}$ giving a clinical appearance of the foot being parallel to the leg. Our patients walked on their heels as the joint gave less support. In five cases we cut the pointed flaps according to simple or plural Z- form plasty. The flaps included the subcutaneous tissue. However, we found that this operation was more indicated in mild contractures.
Trapezoid flap plasty, either the pure type or in combination with free skin transplantation (4 cases), was found to be the best method for creating an active zone (in the ankles) from local tissue. Our operation technique was to divide the sheets of flaps, to fold by longitudinal cut along its crest, and then to cut out trapezoid flaps, starting from the middle of the line or from the line of the joint flexion. The ends of the cuts were given a fork-like form for more complete elimination of tightening and better function of the wound margins with the ends of the grafts. The grafts were displaced towards each other and sutured by touching sides. If tightening were not eliminated completely, another pair of grafts was cut out.

Flaps and sheets of scars that were not included were either removed or used as approaching triangular flaps by plasty. In the follow - up terms the scars became thinner, softer and more elastic due to elimination of tightening. The borders of the replaced flaps became smooth and only slightly marked (Fig.1.).
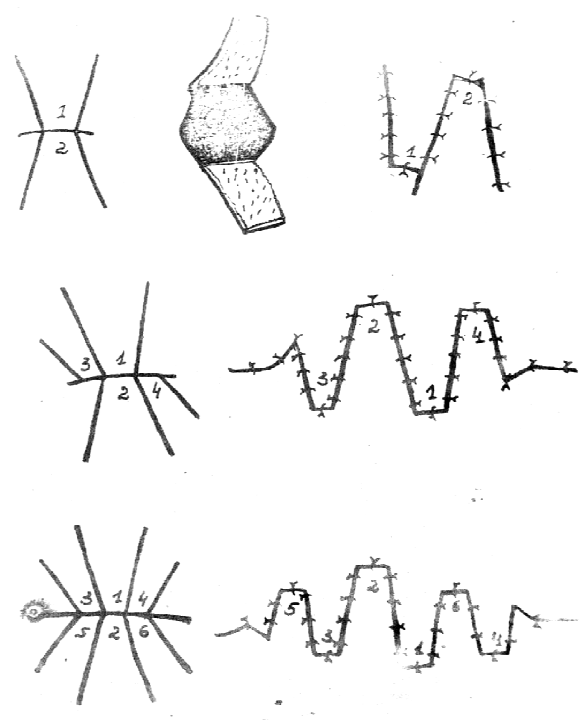

Fig 1. Scheme of treatment for dorsal foot contractures. The forms of two or multiple contrary oriented trapezoid flaps.

\subsection{Lateral Contracture}

In seven cases the scars were located on the lateral surface of the ankle joint, occupying the area of the ankle and can achieve anterior- median line of the joint. The greatest scarring defect was at the margin at the point of transition to healthy skin. It was at the anterior margin that the largest extent of tissues in flexion of the joint occurred. Owing to constant traumatization during the growth of connective tissue, scar contracture continued and as a consequence the margin of the scar protruded, involving healthy skin and forming a told.

The tissue was stored in the thickness of the folds and the scar sheets were deficient in length. This is what caused the contracture.

The best method was routine flap plasty. By practicing a horizontal cut, the scar sheet was separated from healthy 
skin and the scars were cut in direction to the ankle and further passing round it like a fork. We cut full- thickness graft from the healthy skin and used this to close the injury in the scar zone. In this way the graft was full bodied and is grewing together with the youngster, thus preventing relapse of the contracture (Fig.2).
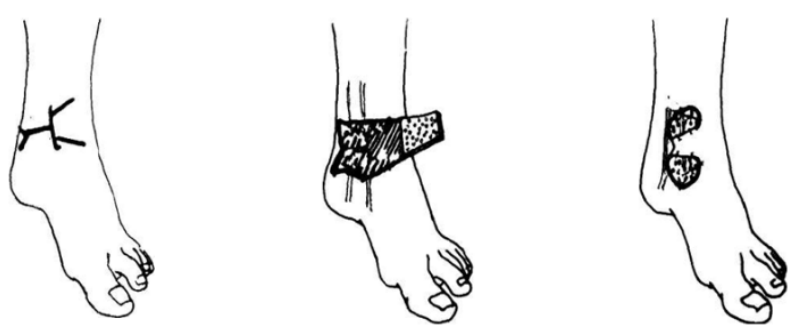

Fig 2. Scheme of treatment for lateral surface scars.

\subsection{Plantiflexion Contractures}

Plantar flexible deformations of the ankle joint were the result of the burn damage of the posterior surface of the shin with involment of the Achilles tendon in the process, leading to the development of eguine foot. When standing or waking, patients with equine foot exert their weight in the anterior section. Out of five cases of keloid scars, one patient had injuries in the zone of the heel tendon and four had suffered deep burns with tissue defect in the affected zone with ulcerous scars.

The operation was performed after the conservative treatment and maturation of scar connective tissue. In such cases, with tissue defects in the area of the tissue of injured heel tendon, we used L- shaped flap plasty from the lateral surface of ankle and foot. We then cut the scars with the ulcer over the tendon. On the lateral side of the distal third of ankle and foot, near the injury we cut the skin-fatty graft and closed the injury and heel tendon from three sides with it. On the wound where we have taken the scrap from we made free transplantation of skin (Fig. 3).
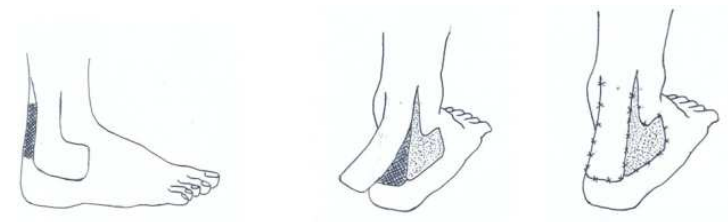

A.

B.

C.

Panel B. L-form skin-fascial graft from the inferior third of the crus and foot has been mobilized.

Panel C. After ulcerating cicatrices dissection the wound was covered by mobilized skin-fascial graft

Fig 3. Panel A. Ulcerating cicatrix in the area of the Achilles tendon zone. The borders of the L-form skin fascial graft have been pointed out.

\subsection{Whole Ankle Joint}

Thick keloid scars do not often develop in the ankle joint area but they may cause dysfunctions and malformations. Scars tighten the joints and cause sharply limited movements ( 7 patients). Such contractures are considered to be the most difficult. It is known that the tighter the contracture is, the more significant is the crooking of the foot to that side.

There is practically no free scar on the whole width, for which reason the chosen method of operation was to cut the scars until full elimination of the contracture, followed by reconstruction of the natural foot position with free splitskin transplantat. As a result skin lining grew, providing good functional and cosmetic outcome

\subsection{Extended contractures of the Dorsum of the Foot (Digits. Ankle Joint)}

The most frequent type of disturbance (59 cases) with significant anatomical variability. The following groups of this disturbance were identified: a) isolated injury to the dorsum of the foot; b) disturbance with extension of scars on the digits causing contractual extension of metatarsalphalangeal joints with or without involvement of ankle joint, with or without syndactylous digits. In terms of surgical rehabilitation, it was important to prevent the development of deformity of the foot bones.

Therefore, at the beginning of subluxation and foot deformity, the operation was performed no later than six months after healing of burn wounds. First of all, functional disorders, distortions, and tightening tissues deforming the foot were eliminated using local tissues (soft scar tissue in the folds, tissue of the transfer). It was shown that the operation must be done in one stage.

All contracting tissues, subluxations, syndactyliae, etc. must be corrected at the same time. Free graft and /or flap plasty was performed, depending upon scar extension, scar thickness, tissue reserve in the folds, and the degree of contracture (Fig.4).

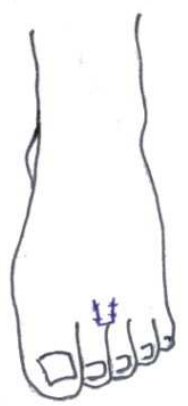

Fig 4. Scheme of treatment of inter-digital symphysis Pi-form.

Patient with large rough scars causing third / fourth degree contractual extension with subluxation of digits and syndactylia had problem with footwear, although the foot static and dynamic functions were not disturbed. In these cases, pathological tissues were incised to the level of metatarsophalangeal joints, thus creating a line of distal incision of dentate form. As this was usually done on the back of the hand, plastic surgery of commissures between the toes was performed using trapezoid, triangular grafts, which were cut out of the surface of inter-digit space or of 
lateral surfaces of digits.

These grafts were fixed with free ends on the level of heads of metatarsal bones. Simultaneously, there was performed redressation, patients' elimination of subluxations and dislocations of digits which were gradually transferred into the position of plantar flexion under the angle from 60 to 900 in metatarsophalangeal joints. The position of the digits were maintained with retrograde Kirshner wires, particularly in III-IV degrees burn contractures. If fixation with wires was not effective, toes were sewed through the nail phalanges by means of thick ligature to the plantar surface of the foot. The technique was effective in eliminating dislocations and subluxations of digits in all patients. Developing wound defects taking in some patients more than $2 / 3$ of the foot dorsum were closed by split - skin transplants $0.30 .4 \mathrm{~mm}$ thick

Results of operations in $71,05 \%$ of all cases the contracture of the foot-ankle joint was completely eliminated and the conditions necessary for the rehabilitation of the injured ankle were ensured. There was improvement after burn treatment or secondary changes due to inflammatory processes and foot inactivity due to its wrong position; in 3,95\% there was not improvement. Negative results were due to deep tissue defect, irreversible changes in the bone- joints, late recourse to medical aid, and post operative effect. We did not observe any ulceration of skin grafts.

\section{Discussion}

Post-burn contractures foot and ankle joint are divided according to their function: flexion and extension. In spite of great years of medical history and research, the anatomy and nature of scar contractures has not been sufficiently studied. Thus, no classification that based on anatomic data has been presented.

Foot and ankle joint contractures are often combined with foot and leg deformities; therefore, more attention is given to foot reconstruction as a more complication. Investigations dedicated solely to post burn ankle scar contractures are an exception. Thus, many aspects of postburn ankle reconstruction remained not researched. An aspect that needs further investigation has to do with the anatomic features of the contractures, the new approach to possibilities of local tissues use, and indications for specific techniques applications.

These results suggest that for treatment of the deformities of ankle-joint induced by burns differential plastic surgery should be used depending on 1) localization and expansion of scars, 2) presence of skin, and 3) presence of bones that are not injured or changed.

The patient with ankle joint must be monitored carefully if there are scars and there is the consequent danger of slow growth of the burned foot joint and development of secondary bone-joint changes. Early surgery is advised depending on the severity of the contracture.
The analysis of burn after-effects and their dynamics in patients as the result of growth of their organism allowed us to determine the contingent of the persons who must be rehabilitated:

- $\quad$ patients with limited deep burn of foot-ankle joint;

- $\quad$ patients with deep burn or burn of the $3 \mathrm{~d}$ degree in foot-ankle joint;

- patients with deep burn even if they have no disjunction of the skeletal muscular apparatus, on discharge from the hospital but having loss of skin surface in the foot-ankle joint.

The experience obtained proves the fact that children of the above mentioned categories should be taken for dispensary for a long period of time until the end of growth (18-19 years) even if there is no initial burn deformities in the foot-ankle joint.

The main role has to be played by rehabilitation centers, where all methods of treatment are available (medical and physical training, ultrasound, kenalogy, radone other medical (pirogenal, lidazi, triamthinolana) and physical treatment (ultrasound, magnetic waves, compression), balneotherapy (radon and hydrogen sulphide bath).

\section{References}

[1] Mikhailov I.A., Popov S.V. Surgical Treatment of the Results of Foot Burns. Paper presented at the International Conference «Intensive Treatment of Severe Burn Patients», Moscow, 1992, 284-286.

[2] Yudenich A.A., Mikhailov I.A., Sarigin P.V. The Surgical Treatment of Post-Burn Scar Deformities of Foot-ankle Joint and Foot. Materials of International Conference «The Actual Problems of Thermic Injury, dedicated to 70th anniversary of S.R.I.», Saint-Petersburg 2002, 461-462.

[3] Mirazimov B.M., Tursunov B.S., Grishkevitch V.M. Postburn Deformities of Extremities in Children. Tashkent: Ibn Sino Publishing House, 1991, 342.

[4] Sakirov B.M. Plastic operation with post burn contractures and deformities of foot and ankle joint. Abstract book. The 14th congress of the International Society for burn Injuries, Canada, 2008; -97.

[5] Shakirov B.M. Sandal Burns and their Treatment in Children. J. Burn Care Rehabilitation. 2004; 25; 501-505.

[6] Shuchanek I., Rihova H., Kaloudova Y., Mager R. Reconstructive Surgeries after Extensive Burns in Children. Acta. Chir. Plast 2004; 45: 139-143.

[7] Schneider J.C. et al. Contractures in burn injury: defining the problems // Burn Care Res. 2006; 27: 508-514.

[8] Mustal T.A. Scars and Keloids. BMJ, 2004; 328:13291330Amarante J., Costa H., Keis J. et al. New distally based fascioculaneous flap of the Ieg // Bril. J. Plast. Surg. - 1986Vol. 39, № 3. 338-340.

[9] Shakirov B.M. Evaluation of different surgical techniques used for correction of post-burn contracture of foot and ankle. J. Annals of Burns and Fire Disasters 2010; 3: 137-143. 
[10] Steinwender G., Saraph V., Zwick E.B., Uitz C, Linhart W. Complex Foot Deformities Associated with Soft-tissue Scarring in Children. J. Foot- Ankle Surg. 2001; 40: 42-49.

[11] Guild S. A new splinting approach for dorsal foot burns. J Burn Care Rehabil. 2001; 22:454-456.

[12] Shakirov B.M., Tursunov B.S., Tagaev K.R. Problems of Rehabilitation of Post-Burn Reconvalecence. J. Allergology and Immunology, Moscow, March 2004, №1, 176.
[13] Leung R.S., Cheng J.S. Burn Contractures of the Foot. J. Foot Ankle 1986; 6: 289-294.

[14] Erdogan B., Gorgu M., Girgin O., Akoz T., Deren O. Application of external fixators in major foot contractures. $\mathrm{J}$ Foot Ankle Surg. 1996; 35:218-221. 